\title{
Análise do comportamento de queima de argilas e formulações para revestimento cerâmico
}

\author{
(Analysis of the firing behavior of clays and formulations \\ for ceramic tiles) \\ M.M.T. Moreno, D. Bartolomeu, R. H. C. Lima \\ DPM-IGCE-UNESP, Av. 24A 1515, Rio Claro, SP 13506-900 \\ mmoreno@rc.unesp.br,danbartolomeu@hotmail.com,humbrtoclima@yahoo.com.br
}

\begin{abstract}
Resumo
O uso de argilas de queima vermelha para a fabricação de placas cerâmicas para revestimentos tornou-se importante devido aos avanços tecnológicos e à qualidade da matéria-prima. Neste processo a moagem é feita por via seca e as temperaturas máximas de queima são maiores que $1000{ }^{\circ} \mathrm{C}$, ocorrendo reações de sinterização via fase líquida. Este trabalho aborda diversos aspectos que envolvem a queima deste tipo de argilas e suas misturas visando obter as melhores condições de sinterização. As argilas individuais foram analisadas usando os seguintes parâmetros: \% em peso na composição, temperatura máxima de queima e absorção de água. Corpos-de-prova foram queimados em laboratório, em temperaturas de 800 a $1100{ }^{\circ} \mathrm{C}$, sendo que depois da caracterização das propriedades individuais, combinações binárias e ternárias foram processadas nas mesmas condições e analisadas. Os resultados obtidos permitiram elaborar uma proposta para preparar misturas com base nas características das matérias-primas. Devido a que as combinações apresentam variações, que dependem principalmente da distribuição do tamanho das partículas e conteúdo de fundentes, é necessário fazer correções na composição para alcançar as propriedades desejadas, o que é facilitado com a metodologia proposta.
\end{abstract}

Palavras chave: argilas vermelhas, revestimentos cerâmicos, comportamento térmico.

\begin{abstract}
The use of red fired clays for the production of ceramic tiles has become important due to the technological progress and the improvement of raw material quality. In this processing, dry milling is used and the maximum firing temperatures are larger than $1000{ }^{\circ} \mathrm{C}$ with the occurrence of sintering reactions through liquid phase. In this work, the behavior of red clays and their mixtures to obtain the best sintering conditions was studied. Individual types of clay were analyzed using the following parameters: wt.\% in the composition, maximum firing temperature and absorption of water of the individual raw materials. Test specimens were fired in laboratory with temperature varying between 800 and $1100{ }^{\circ} \mathrm{C}$. After characterization of the individual properties, binary and ternary combinations of clays were analyzed using the same conditions of the individual ones. The results obtained allowed us to elaborate a proposal of how to prepare these types of mixtures based on the raw material. Because mixtures show variations on their response that are mainly dependent on the particle size distribution and fluxing compounds, it is necessary to perform corrections in the composition of the mixture to reach the desired properties. Using the proposed methodology, it is possible to orientate the changes in the composition in order to reach the desired result.
\end{abstract}

Keywords: red clays, ceramic tiles, firing behavior.

\section{INTRODUÇÃO}

O principal produto no pólo de Santa Gertrudes são as placas cerâmicas para revestimento preparadas por prensagem de argila moída a seco, onde os corpos cerâmicos são submetidos a ciclos de queima rápida (entre 20 e $30 \mathrm{~min}$ ) com temperatura máxima acima de $1000{ }^{\circ} \mathrm{C}$. Nessas temperaturas os mecanismos de sinterização estão condicionados à formação de uma fase líquida capaz de proporcionar as propriedades requeridas conforme a classificação do produto [1], sendo necessário adequar um programa de queima à argila (ou mistura de argilas) em questão. Normalmente o procedimento é feito através de testes empíricos que dependem muito da experiência e não do conhecimento da matéria-prima, a qual muitas vezes não aparenta diferenças significativas ao longo do perfil, o que nem sempre é verdadeiro.

As argilas são extraídas basicamente da Formação Corumbataí, onde o perfil de sedimentos apresenta variação no grau de alteração aumentando da base para o topo devido a processos de hidrotermalismo e ação intempérica [2, 3], conseqüentemente a mineralogia e a distribuição granulométrica obtida no processo de moagem também variam. A distribuição granulométrica que resulta da moagem é importante no empacotamento das partículas durante a prensagem, da mesma forma que na evolução da sinterização, uma vez que está relacionada 
parcialmente à quantidade de contatos inter-granulares. $\mathrm{O}$ argilomineral dominante geralmente é a illita, existindo em todo o perfil [4], encontrando-se associada a outros, como cloritas, principalmente nos níveis basais, e argilominerais expansivos nos níveis mais expostos ao intemperismo químico. A caulinita pode estar presente nos níveis mais superficiais [5]. Em termos de composição, a principal característica que define o bom comportamento das argilas da Formação Corumbataí é sua capacidade de formar fase líquida a temperaturas relativamente baixas, contribuindo para isso o teor de elementos alcalinos contidos nos feldspatos (dominantemente sódicos e de granulometria fina) e na matriz illítica que disponibiliza o potássio para as reações de sinterização. O conjunto de componentes destas argilas constitui uma massa cerâmica "quase pronta", fato que contribui para obter produtos baratos e de boa qualidade, sendo necessário preparar formulações que associem as propriedades fundentes de algumas argilas (normalmente duras), com outras que desagregam mais facilmente durante a moagem a seco (mais alteradas) gerando grãos mais finos, de tal forma que a distribuição granulométrica obtida, resulte num empacotamento satisfatório durante a prensagem.

A dificuldade está no controle de qualidade dos produtos causada pelas variações mineralógicas e grau de alteração que as rochas apresentam, tanto na direção vertical como na horizontal. Esta situação gera problemas tais como queda na produção, geração de rejeitos, extração inadequada da matéria-prima, danos ao meio ambiente, entre outros, sendo que para diminuir estes efeitos indesejáveis é necessário conhecimento das características das matérias-primas e suas combinações.

A proporção de cada argila usada na preparação das massas é determinada empiricamente, necessitando correções quando as variações se manifestam, as quais são aplicadas principalmente na massa, na temperatura e no tempo de queima. Neste trabalho foram estudados diferentes aspectos que envolvem a sinterização de argilas, com base nas propriedades cerâmicas adquiridas após queima e suas variações com a temperatura, visando entender o comportamento apresentado pelas argilas analisadas, selecionadas em três diferentes minas, sendo duas da base (duras) e duas do topo dos perfis. A partir destas observações foi estudado o comportamento de queima de misturas binárias e ternárias (dessas argilas) o que originou uma proposta de formulação de massas de dois ou mais componentes, que permite ajustes direcionados na formulação.

\section{EXPERIMENTAL}

Seleção e coleta de matérias-primas com características diferenciadas de dureza, refratariedade, localização e aspecto físico, de acordo com conhecimento prévio das mesmas. Estas tiveram como fonte as minas Pieroni (amostras P1 e P2), Granusso (amostra G) e Cruzeiro (amostra CR); Tratamento das amostras: secagem em estufa $\left(60{ }^{\circ} \mathrm{C}\right)$, moagem em moinho de martelos de laboratório com peneira de $1 \mathrm{~mm}$ e peneiramento da porção usada para prensagem dos corpos-de-prova em malha de $500 \mu \mathrm{m}$ de abertura.

Caracterização granulométrica, química e mineralógica das amostras: 1- determinação da distribuição granulométrica de moagem usando peneiras de abertura de 500 a $53 \mu \mathrm{m}$ e a úmido peneiras de abertura 45,38 e $25 \mu \mathrm{m}$; 2- análise química por espectrometria de fluorescência de raios $\mathrm{X}$ (FRX) em aparelho Philips PW2510 e mineralógica por difração de raios X (DRX), Siemens D5000, com radiação $\mathrm{Cuk}_{\alpha}$, filtro de Ni e varredura $3^{\circ}(2 \theta) / \mathrm{min}$.

Propriedades físicas das peças cerâmicas: 1- umidificação e prensagem de corpos-de-prova em molde de 7 × $2 \mathrm{~cm}$ (aproximadamente $19 \mathrm{~g}$ ) com umidade de $8 \%$ e pressão suficiente para obter densidade de $2{\mathrm{~g} . \mathrm{cm}^{-3}}^{-3}$ visando as mesmas condições das massas formuladas com as matériasprimas estudadas. Esses valores de densidade e umidade são usados na indústria cerâmica do pólo cerâmico da região de Santa Gertrudes; 2- secagem a $110^{\circ} \mathrm{C}$ e queima em forno Maitec FE50RP (800, 900, 950, 1000, 1020, 1040,1060, 1080,1090 e $1100{ }^{\circ} \mathrm{C}$ ), em ciclos de queima rápida com taxas de aquecimento de $15^{\circ} \mathrm{C} / \mathrm{min}$ e 4 patamares de $2 \mathrm{~min}$; 3- obtenção das propriedades cerâmicas [6] priorizando a retração linear de secagem (RLS), retração linear de queima (RLQ) e absorção de água (AA).

Procedimento para preparação de mistura: 1- selecionar uma temperatura $(\mathrm{T})$ de queima e um valor de absorção de água (AA) para o produto requerido; 2- selecionar um número $\mathrm{N}$ de amostras que se deseja misturar; 3processar cada uma delas nas mesmas condições (secar, moer, peneirar, umidificar, prensar, secar, queimar, etc.), obtendo um total de 3 a 5 peças por amostra, dependendo do tamanho; 4- queimar na temperatura $\mathrm{T}$; 5- determinar a absorção de água, AA, de cada uma; 6- selecionar três argilas (inicialmente) com valores diferentes de AA de tal maneira que o valor desejado se localize dentro do intervalo de duas combinações binárias entre as três argilas; 7- marcar os nomes das amostras nos vértices de um triângulo (com escala nos lados para as respectivas proporções das misturas); 8- calcular as proporções de cada argila a partir do valor de AA desejado e dos respectivos componentes para duas combinações de argila (duas combinações argilas devem conter o valor de AA que se deseja obter), conforme exemplo descrito em Resultados; 9- se a mistura a ser testada for de 2 componentes unicamente, preparar corpos de prova com as proporções encontradas e modificar estas deslocando o ponto (aumentando ou diminuindo a proporção dos componentes, conforme o resultado da AA obtido para cada componente (usar somente o lado correspondente no triângulo); 10- para amostras de três componentes, continuar o procedimento; 11- calcular mais duas proporções para AA diferentes da calculada no item 8 selecionando um valor acima e outro abaixo; 12- marcar no triângulo (no respectivo lado); 13- unir os dois pontos para a mesma AA; 14- selecionar pontos de teste ternários dentro do triângulo (proporções das argilas individuais) para a absorção de água desejada (item 8); 15- processar nas mesmas condições que os corpos de prova das argilas individuais e medir a AA; 16- modificar a composição deslocando o ponto dentro do gráfico conforme 
seja conveniente com base nas outras linhas de AA; 17 para fazer misturas com mais de três materiais, é necessário modificar os componentes nos vértices do triângulo, colocando misturas preparadas e testadas previamente.

\section{RESULTADOS}

\section{Descrição das amostras}

As quatro amostras de argila (Tabela I) foram selecionadas visando fornecer informações sobre diferenças no comportamento de queima e conseqüentemente nas propriedades cerâmicas, bem como mineralógicas e químicas, para entender os mecanismos envolvidos no processo de queima. As amostras correspondem a níveis individuais de frentes de lavra com características diferentes. As duas argilas da mina Pieroni (P1 e P2) pertencem ao topo [7] e apresentam diferenças significativas nas suas características, estando P2 (de cor amarelada) localizada logo abaixo do solo e P1 (de cor roxa) abaixo de P2. Estas argilas não são atualmente utilizadas pela indústria cerâmica, uma vez que não possuem os requisitos das matérias-primas para revestimentos. As amostras $\mathbf{G}$ e $\mathbf{C R}$ pertencem à porção inferior das minas Granusso e Cruzeiro, respectivamente, e são conhecidas como argilas duras, sendo que $\mathbf{G}$ (siltito argiloso roxo maciço) está localizada na base da coluna estratigráfica [8] e CR (siltito variegado) a uma altura de aproximadamente $13 \mathrm{~m}$ da base, de um total de $44 \mathrm{~m}$ [9].

Tabela I - Umidade das massas e densidade após a prensagem e secagem dos corpos-de-prova.

[Table I - Humidity of the mass, dry density and green density of test specimens.]

\begin{tabular}{ccccc}
\hline amostra & Mina & $\begin{array}{c}\text { Umidade } \\
(\%)\end{array}$ & $\begin{array}{c}\text { Dp } \\
\left(\mathrm{g} . \mathrm{cm}^{-3}\right)^{\mathrm{a}}\end{array}$ & $\begin{array}{c}\text { Ds } \\
\left(\mathrm{g} . \mathrm{cm}^{-3}\right)^{\mathrm{b}}\end{array}$ \\
\hline P1 & Pieroni & 7,9 & $2,00 \pm 0,01$ & $1,88 \pm 0,02$ \\
G & Granusso & 7,9 & $2,00 \pm 0,01$ & $1,84 \pm 0,02$ \\
CR & Cruzeiro & 8,0 & $2,00 \pm 0,01$ & $1,85 \pm 0,01$ \\
P2 & Pieroni & 8,2 & $1,90 \pm 0,01$ & $1,76 \pm 0,01$ \\
\hline
\end{tabular}

${ }^{a}$ densidade de prensagem, ${ }^{b}$ densidade a seco.

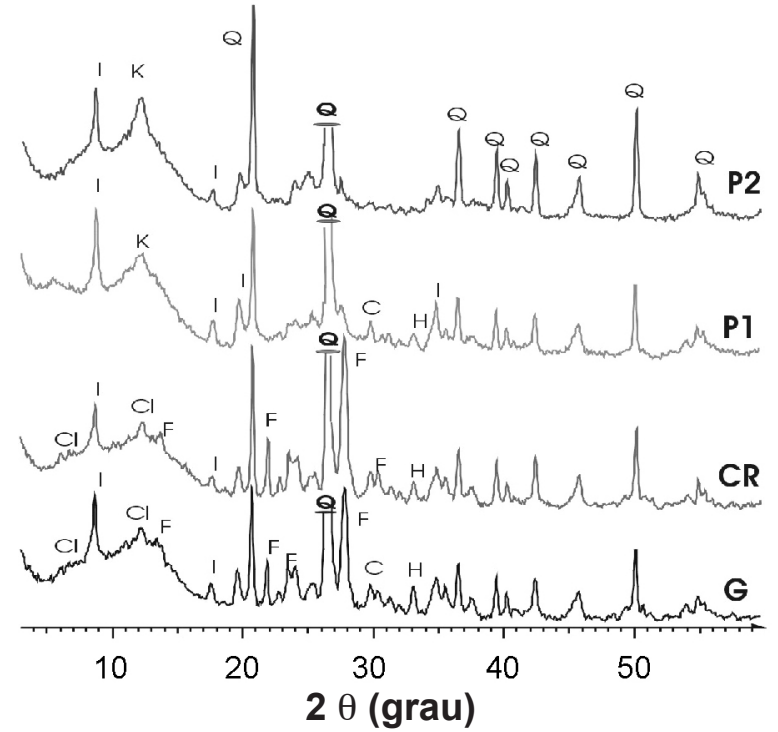

Figura 1: Difratogramas de raios $\mathrm{X}$ de amostra total para $\mathrm{P} 2, \mathrm{CR}$, G e P1, mostrando posições de clorita (CL), caulinita (K), quartzo (Q), Feldspato sódico (F), Calcita (C) e Hematita (H).

[Figure 1: X-Ray diffraction pattern for bulk sample from $P 2, C R$, $G$ and $P 1$ showing positions of chlorite (CL), Kaolinite (K), quartz (Q), Na-Feldspar $(F)$ calcite $(C)$ and hematite $(H)$.]

A Tabela I apresenta as condições de prensagem das amostras, sendo que $\mathbf{P 2}$ não atingiu a densidade escolhida para a preparação das misturas devido à distribuição granulométrica inadequada, fato que se reflete no comportamento de queima apresentado, significando que sua entrada nas formulações pode trazer desvios grandes em relação aos valores esperados, a menos que a argila associada compense as deficiências na distribuição granulométrica, de tal forma que melhore significativamente o empacotamento.

\section{Caracterização química e mineralógica}

A composição química das amostras de argila é mostrada na Tabela II e proporciona um suporte para a análise mineralógica obtida por difração de raios $X$ mostrada na Fig. 1, onde o pico principal do quartzo foi cortado para visualizar melhor os outros minerais. As quatro amostras

Tabela II - Composição química das amostras de argila em \% de óxido.

[Table II - Chemical composition of clay samples in wt.\% of oxide.]

\begin{tabular}{cccccccccccc}
\hline Amostra & $\mathrm{SiO}_{2}$ & $\mathrm{TiO}_{2}$ & $\mathrm{Al}_{2} \mathrm{O}_{3}$ & $\mathrm{Fe}_{2} \mathrm{O}_{3}$ & $\mathrm{MnO}$ & $\mathrm{MgO}$ & $\mathrm{CaO}$ & $\mathrm{Na}_{2} \mathrm{O}$ & $\mathrm{K}_{2} \mathrm{O}$ & $\mathrm{P}_{2} \mathrm{O}_{5}$ & L.O.I. $^{\mathrm{a}}$ \\
\hline $\mathrm{P} 1$ & 64,55 & 0,55 & 16,28 & 5,99 & 0,03 & 3,01 & 0,07 & 0,67 & 2,62 & 0,07 & 5,43 \\
$\mathrm{G}$ & 67,86 & 0,63 & 14,39 & 5,21 & 0,03 & 1,97 & 0,62 & 2,63 & 3,55 & 0,19 & 2,90 \\
$\mathrm{CR}$ & 69,28 & 0,54 & 13,97 & 5,45 & 0,02 & 1,64 & 0,45 & 3,11 & 3,22 & 0,11 & 2,27 \\
$\mathrm{P} 2$ & 70,94 & 0,50 & 14,52 & 5,34 & 0,01 & 2,17 & 0,01 & 0,01 & 1,41 & 0,04 & 5,04 \\
\hline
\end{tabular}

aperda ao fogo. 
contém argilominerais da família das illitas (I), principal fonte de potássio, sendo que nas amostras da base (CR e G), o teor de $\mathrm{K}_{2} \mathrm{O}$ é maior. Os gráficos apresentam uma série de picos mal definidos entre as posições 10 e $7 \AA$ e acima de $14 \AA$ os quais correspondem a estruturas estratificadas de argilominerais [9], que são componentes intermediários resultantes da alteração intempérica/hidrotermal nos perfis de argila. $\mathrm{O}$ teor de $\mathrm{K}_{2} \mathrm{O}$ em $\mathbf{P 2}$ é o menor entre as quatro amostras e os elementos mais móveis, $\mathrm{Ca}$ e $\mathrm{Na}$, estão praticamente ausentes. Para isto contribuem sua localização na parte superior e a maior permeabilidade devido ao maior conteúdo de quartzo livre conforme a análise química e de difração de raios $\mathrm{X}$. O teor maior de $\mathrm{MgO}$ nas amostras do topo se deve provavelmente aos compostos intermediários (interestratificados) e óxidos hidratados não cristalinos.

P1 contém principalmente quartzo, illita e argilominerais do grupo da caulinita, sendo estes responsáveis pelo maior conteúdo de $\mathrm{Al}_{2} \mathrm{O}_{3}$ (Tabela II ). Tanto em P1 como em P2 foi detetada a presença discreta de feldspatos, os quais sofreram alteração intempérica devido a sua localização na porção superior da frente de lavra, resultando em maior perda ao fogo devida à presença de minerais hidratados e hidróxidos metálicos não cristalinos. G e CR, amostras da base, apresentam feldspato sódico e argilominerais cloríticos, além de illita e quartzo, preservados devido à menor exposição intempérica. Em $\mathbf{C R}$ o conteúdo de $\mathrm{Na}_{2} \mathrm{O}$ é maior que em $\mathbf{G}$ confirmando seu maior conteúdo em feldspatos (dominantemente sódicos), como observado pela altura dos picos nos difractogramas; Nas argilas vermelhas foi identificada a hematita, menos em $\mathbf{P 2}$ (amarelada), onde também a calcita parece estar ausente. Nas outras $(\mathbf{G}, \mathbf{C R}$ e P1) foi identificada calcita do tipo magnesiana

\section{Tamanho de partículas}

A distribuição de tamanho de partículas após a moagem (Fig. 2) foi realizada por peneiramento a seco entre $0,5 \mathrm{e}$ $0,053 \mathrm{~mm}$ e a úmido (devido a dificuldades de peneiramento) nas peneiras de $0,045,0,038$ e $0,025 \mathrm{~mm}$ visando estimar a influencia da distribuição do tamanho das partículas no empacotamento durante a prensagem dos corpos-de-prova. As amostras da porção basal ( $\mathbf{G}$ e $\mathbf{C R})$ possuem distribuição semelhante até $0,075 \mathrm{~mm}$ diferenciando-se nas frações com tamanho $<0,075 \mathrm{~mm}$, onde $\mathbf{G}$ tende a maior conteúdo de finos. Por serem duras, a granulometria que prevalece é a da moagem, diferenciando-se conforme diminui o tamanho das partículas em função do grau de alteração e composição mineralógica. Como foi visto acima (Fig. 1) estas amostras possuem um conteúdo maior de feldspatos, principalmente a CR. Nas amostras da porção superior das minas, há uma forte influencia da graulometria natural durante a moagem (a argila desagrega mais facilmente), $\mathbf{P 2}$ mostra pouca variação no tamanho das partículas causando maior porosidade durante o empacotamento, nestas condições, os corpos-de-prova desta

Tabela III - Peso (\%) de partículas retidas em malhas ABNT de 0,500 a 0,025 mm.

[Table III - Weight (\%) of particles detain in sieve ABNT (0.500 to $0.025 \mathrm{~mm})$.]

\begin{tabular}{llllllllllll}
\hline Amostra & 0,500 & 0,355 & 0,180 & 0,125 & 0,090 & 0,075 & 0,053 & 0,045 & 0,038 & 0,025 & $<0,025$ \\
\hline P1 & 0,79 & 3,27 & 13,11 & 8,21 & 6,78 & 2,54 & 6,88 & 0,80 & 3,12 & 3,36 & 51,15 \\
G & 1,43 & 5,96 & 21,60 & 10,01 & 5,66 & 3,07 & 4,20 & 2,50 & 4,09 & 2,14 & 39,54 \\
CR & 1,87 & 5,60 & 21,67 & 9,76 & 5,74 & 3,21 & 7,51 & 2,06 & 4,87 & 2,71 & 35,00 \\
P2 & 0,71 & 1,46 & 8,16 & 5,91 & 5,35 & 3,56 & 6,99 & 2,25 & 5,18 & 5,66 & 54,77 \\
\hline
\end{tabular}

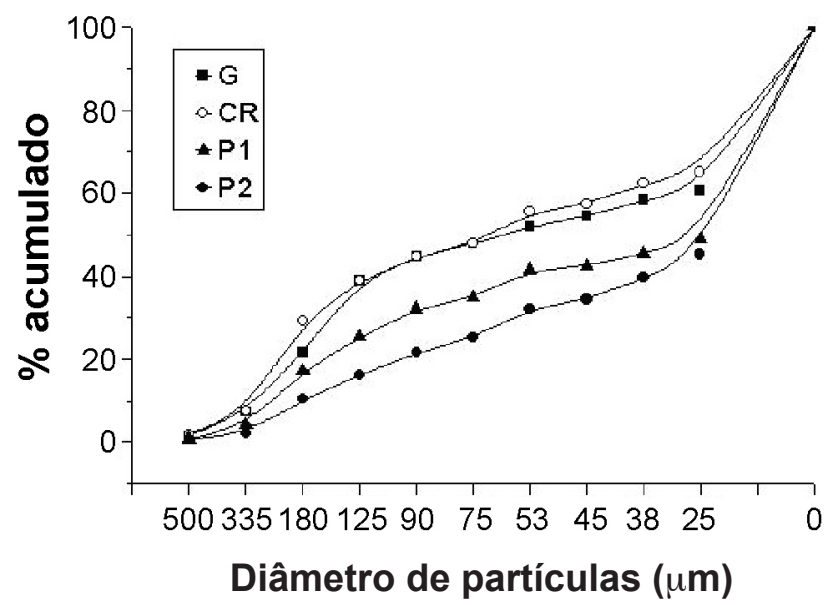

Figura 2: Distribuição de tamanho de partículas após moagem na faixa de 0,500 a 0,025 mm. [Figure 2: Particle size distribution after milling varying into a range from 0.500 to $0.025 \mathrm{~mm}$.] 
amostra não atingiram a densidade de $2,0 \mathrm{~g} / \mathrm{cm}^{3}$ das outras amostras. Já a argila $\mathbf{P 1}$ tem menor teor de partículas grossas e médias que $\mathbf{G}$ e $\mathbf{C R}$ significando que a diferença de massa corresponde a partículas menores que $0,025 \mathrm{~mm}$ (Tabela III), resultando em melhor acomodamento das partículas durante a secagem dos corpos-de-prova, isto fica evidente quando se comparam os valores de retração linear de secagem (RLS) de P1, G, CR e P2, respectivamente $0,24 \pm 0,03,0,05 \pm 0,03$, $0,03 \pm 0,02$ e $0,02 \pm 0,01$.

\section{Sinterização}

Os estudos realizados neste trabalho foram baseados nos dados de absorção de água (AA). Estes são mostrados nos gráficos da Fig. 3, onde se procurou traçar as tendências de variação ditadas pelos mecanismos de sinterização dominantes nas respectivas faixas de temperatura. Notase na figura que o número de etapas para cada argila é o mesmo, variando na faixa de temperatura e na velocidade da sinterização conforme mostra a inclinação dos respectivos segmentos de reta. Em temperaturas baixas ocorrem reações de pré-sinterização como desidratação, desidroxilação de argilominerais, decomposição de matéria orgânica e carbonatos, oxidação, etc., produzindo transformações que posteriormente, em temperaturas mais altas, podem formar fases estáveis [10].

Em um pó compactado de alumina combinada com outros componentes, de maneira a formar fase líquida, a evolução da compactação mostra uma taxa de sinterização maior numa etapa intermediária onde há fase fluída suficiente para umedecer os grãos, a qual continua enquanto houver condições de solubilização da fase sólida [11]. Este comportamento pode ser comparado ao observado nas argilas estudadas onde, no estágio inicial (Fig. 3), a retração é pequena e as reações ocorrem dominantemente por difusão sólida, seguido da etapa 2 onde ocorre uma forte contração nas peças, causada por reações via fase líquida, e da etapa 3 onde há vitrificação e a absorção de água atinge o menor valor [12], com posterior liberação de gases que provocam uma nova expansão, para os quais contribuem as reações de decomposição dos feldspatos que podem conter água no interior das partículas [13] e a redução de parte do ferro pelo carbono residual presente no sistema [9]. Contribuem para a compactação, na presença de fase líquida, a dissolução de arestas e pequenas partículas; rotação de partículas angulares para melhor acomodação; alta difusão através do líquido, podendo ocorrer cristalização em outra região produzindo crescimento dos grãos; penetração rápida do líquido entre os grãos e dispersão de agregados; também o fluído viscoso no limite dos grãos pode acomodar tensões entre regiões microscópicas com pequenas diferenças na taxa de retração [11]. Os mecanismos de sinterização ativos em cada etapa se refletem ao analisar a variação das propriedades cerâmicas com a temperatura conforme ilustrado na Fig. 3.

As amostras $\mathbf{G}$ e $\mathbf{P 1}$ têm comportamento mais próximo entre si, porém o maior teor de fundentes de $\mathbf{G}$ faz com que os gráficos de AA se cruzem na etapa 2, entretanto na etapa
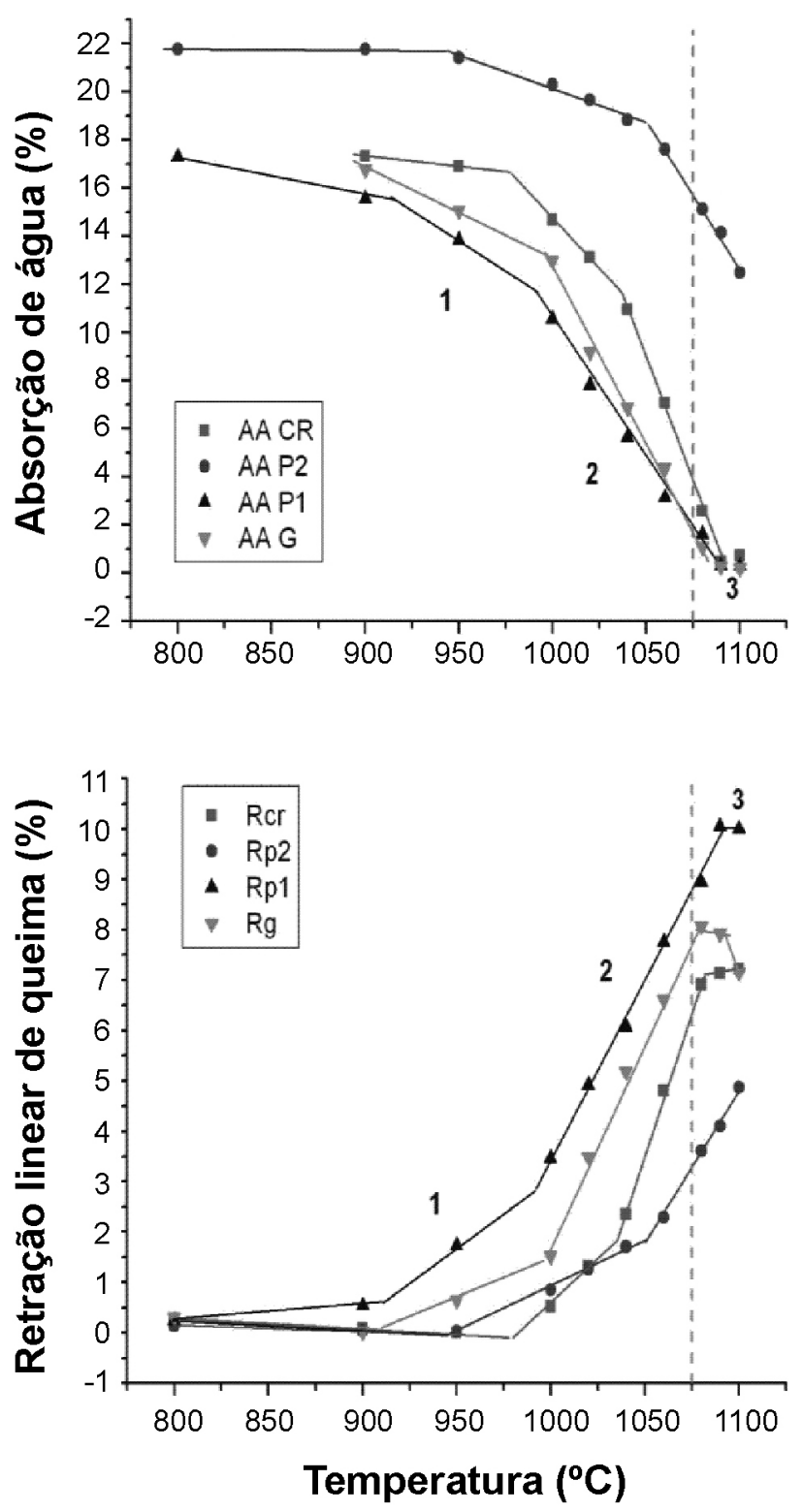

Figura 3: Absorção de água e retração linear de queima para as 4 amostras estudadas.

[Figure 3: Water absorption and linear firing shrinkage of the studied samples.]

1 a maior inclinação da reta é de $\mathbf{P 1}$, indicando sinterização mais eficiente por difusão sólida devido ao maior número de contatos entre as partículas, proporcionado pelo maior conteúdo de finos que preenche os espaços intergranulares. As amostras $\mathbf{G}$ e $\mathbf{C R}$ possuem um alto teor de fundentes, porém, $\mathbf{C R}$ tem maior proporção de grãos grossos o que retarda o início da sinterização [11] em relação a $\mathbf{G}$ nas etapas 1 e 2, porém, uma vez iniciada, se processa mais rápido, influenciada pelo maior teor de feldspatos alcalinos que, associados à illita, desenvolvem taxas de reação maiores. A amostra P2, nas temperaturas ensaiadas, devido ao menor empacotamento $\left(1,76 \mathrm{~g} . \mathrm{cm}^{-3}\right.$ de densidade a seco, sendo de aproximadamente $1,85 \mathrm{~g} . \mathrm{cm}^{-3}$ para as outras amostras, conforme a Tabela I) e baixo teor de elementos alcalinos, 
não atingiu o limite de retração (Fig. 3), apresentando, no intervalo de sinterização 2, variação semelhante com P1 (são quase paralelas), uma vez que esta amostra também tem poucos elementos alcalinos se comparada com $\mathbf{G}$ e $\mathbf{C R}$, sendo a diferença principal, o empacotamento das partículas que provoca um atraso em P2.

Absorção de água (AA) como base de cálculo para preparação de misturas

A mistura de duas argilas com a mesma distribuição granulométrica nem sempre apresenta propriedades físicas correspondentes à soma das frações com que cada componente participa, uma vez que os componentes e a quantidade relativa das mesmas é que determina o comportamento durante a queima (desidratação, liberação de gases, oxidação e redução de componentes/contaminantes, desenvolvimento de eutéticos, estabilidade e cristalização de novas fases, etc.), principalmente nas temperaturas em que a sinterização é dominada por reações via fase fluída. No caso das argilas estudadas nem a composição nem a granulometria de moagem são constantes, fato que introduz variações nas massas, sendo necessário manter um controle sobre as mesmas. Dados aproximados podem ser obtidos a partir dos componentes, fazendo correções quando necessário, para manter os produtos dentro de uma faixa de tolerância.

Para uma mistura de dois componentes, queimada a uma temperatura Tm, e cujas frações na massa são $X_{1}$ e $X_{2}$, esperase, em principio, que a absorção de água resulte em um valor localizado entre as AA dos componentes individuais (AA1 e AA2), permitindo calcular a proporção de cada argila mediante as seguintes relações:

$$
\begin{aligned}
& \mathrm{AAm}=\mathrm{X}_{1} \mathrm{AA} 1+\mathrm{X}_{2} \cdot \mathrm{AA} 2 \\
& \mathrm{X}_{1}+\mathrm{X}_{2}=1 \\
& \mathrm{X}_{2}=1-\mathrm{X}_{1}
\end{aligned}
$$

Substituindo (C) em (A)
Tabela V - Variação da absorção de água com a temperatura para amostra F4R: valores calculados e experimentais.

[Table V - water absorption behavior with the firing temperature for the sample F4R: calculated and experimental values.]

\begin{tabular}{ccc}
\hline Formulação & $\begin{array}{c}\text { AAcal }^{\mathrm{a}} \\
(\boldsymbol{\%})\end{array}$ & $\begin{array}{c}\text { AAex }^{\mathrm{b}} \\
(\boldsymbol{\%})\end{array}$ \\
\hline F4R_1035 & 9,8 & 9,6 \\
F4R_1045 & 8,1 & 8,4 \\
F4R_1055 & 6,3 & 7,1 \\
F4R_1060 & 5,7 & 6,5 \\
F4R_1080 & 2,4 & 3,8 \\
\hline
\end{tabular}

a absorção de água calculada,

${ }^{b}$ absorção de água experimental.

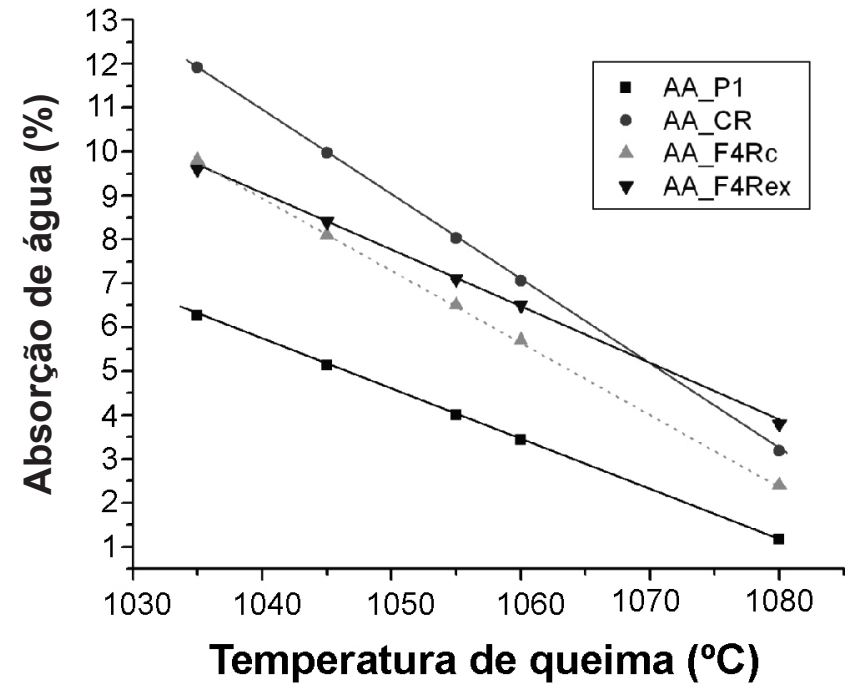

Figura 4: Absorção de água da mistura binária F4R calculada com os valores de AA das argilas individuais e os resultados experimentais.

[Figure 4: Absorption of water of the calculated and experimental binary mixture F4R.]

Tabela IV - Absorção de água e retração linear de queima para misturas de dois componentes calculadas e testadas experimentalmente.

[Table IV - Modeled and experimental values of water absorption and linear firing shrinkage of two components mixtures.]

\begin{tabular}{ccccccc}
\hline Amostra & $\mathrm{T}\left({ }^{\circ} \mathrm{C}\right)$ & $\begin{array}{c}\mathrm{X}_{\mathrm{P} 1-} \mathrm{X}_{\mathrm{G}-} \\
\mathrm{X}_{\mathrm{CR}}\end{array}$ & $\begin{array}{c}\mathrm{AAex}^{\mathrm{a}} \\
(\%)\end{array}$ & $\begin{array}{c}\mathrm{AAcal}^{\mathrm{b}} \\
(\%)\end{array}$ & $\begin{array}{c}\mathrm{RLQex}^{\mathrm{c}} \\
(\%)\end{array}$ & $\begin{array}{c}\text { RLQcal }^{\mathrm{d}} \\
(\%)\end{array}$ \\
\hline F1R & 1050 & $00 \_74 \_26$ & 7,8 & 6,0 & 3,5 & 5,2 \\
F2R & 1050 & $56 \_00 \_44$ & 7,0 & 6,0 & 4,6 & 5,8 \\
F3R & 1055 & $00 \_51 \_49$ & 8,0 & 6,0 & 3,3 & 5,2 \\
F4R & 1055 & $38 \_00 \_62$ & 7,1 & 6,0 & 4,2 & 5,3 \\
\hline
\end{tabular}

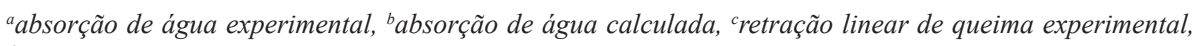
${ }^{d}$ retração linear de queima calculada. 


$$
\mathrm{X}_{1}=(\mathrm{AAm}-\mathrm{AA} 2) /(\mathrm{AA} 1-\mathrm{AA} 2)
$$

Com os valores de $\mathrm{X}$ para cada componente de uma mistura, os valores aproximados de outras propriedades cerâmicas podem ser calculados. Da mesma forma, outras propriedades dos componentes podem servir de base para calcular as frações da mistura. Neste trabalho foi escolhida a absorção de água por ser uma propriedade usada para classificação das peças cerâmicas e os erros de medição são menores quando comparados, por exemplo, com a retração linear de queima (RLQ), embora este seja um valor que pode ser obtido mais rapidamente.

\section{Misturas de dois componentes}

Foram preparadas quatro combinações binárias com a finalidade de comparar os dados calculados com os experimentais (Tabela IV). Foi excluída a amostra P2 devido a que, os corpos-de-prova desta amostra, não atingiram a densidade de 2,0, como requerido para as misturas, eliminando assim uma possível interferência.

Todas as combinações resultaram em AA maior que a esperada (Tabela IV), sendo que as P1_CR tiveram valores mais próximos devido ao teor maior de finos de P1, que permite melhor empacotamento das partículas aumentando o número de contatos entre as mesmas. Nas combinações das duas amostras duras $\mathbf{G}$ e CR (F1R e F3R) a AA ficou mais próxima de $\mathbf{C R}$, faltando grãos finos para preencher os vazios intergranulares. $\mathrm{O}$ aumento de cinco graus na temperatura de F3R em relação a F1R não mostrou mudança apreciável, devido a que F3R tem mais $\mathbf{C R}$, portanto maior proporção de grãos grossos.

A variação da AA com a temperatura (entre 1035 e $\left.1080{ }^{\circ} \mathrm{C}\right)$ para a formulação F4R $(38 \% \mathrm{P} 1$ e $62 \% \mathrm{CR})$ é mostrada na Tabela $\mathrm{V}$ e na Fig. 4. Conforme aumenta a temperatura há uma tendência de aproximação às características de $\mathbf{C R}$ pura, chegando a piorar acima de $1070{ }^{\circ} \mathrm{C}$ devido a que o teor de fundentes da mistura é menor que em $\mathbf{C R}$, e o empacotamento obtido não foi satisfatório. Neste caso, um aumento da temperatura não aproxima os valores experimentais daqueles calculados, sendo melhor solução um aumento na proporção de P1 ou moagem mais fina de CR. Se o resultado fosse oposto (AAexp.< AA de P1), uma redução da temperatura seria uma alternativa de correção interessante por diminuir os gastos com energia. Quando o valor experimental está entre os dos componentes, ajuste nas proporções pode ser mais conveniente, dependendo da disponibilidade da matéria-prima.

\section{Mistura de três componentes}

Para este tipo de combinação é necessário um diagrama triangular, contendo em cada vértice um componente ou uma mistura, como mostra a Fig. 5 para formulações P1_G_ CR. Neste caso, as possibilidades de combinação são mais abrangentes visando um mesmo valor de AA calculado e a construção das linhas de AA constante é feita nas laterais do triângulo, da mesma forma que foi feito para as combinações binárias conforme exemplificado abaixo:

Exemplo de cálculo de massas

Dados:Argilas (matérias-primas): A, B e C

Absorção de água: AAm (requerida p/mistura); $\mathrm{AA}_{\mathrm{A}}$, $\mathrm{AA}_{\mathrm{B}}, \mathrm{AA}_{\mathrm{C}}$

$\mathrm{AAm}=6,5 ; \mathrm{AA}_{\mathrm{A}}=7,5 ; \mathrm{AA}_{\mathrm{B}}=4 ; \mathrm{AA}_{\mathrm{C}}=5,5$

Cálculos:

Combinação A_B

$\mathrm{X}_{\mathrm{A}}=\left(\mathrm{AAm}-\mathrm{AA}_{\mathrm{B}}\right) /\left(\mathrm{AA}_{\mathrm{A}}-\mathrm{AA}_{\mathrm{B}}\right) ; \mathrm{X}_{\mathrm{B}}=1-\mathrm{X}_{\mathrm{A}} ; \mathrm{X}_{\mathrm{A}}=0,71 ;$ $\mathrm{X}_{\mathrm{B}}=0,29 ; \mathrm{A}=71 \% ; \mathrm{B}=29 \%$

Note-se que a combinação das argilas B e C não satisfaz a condição de $\mathrm{AA}=6,5$, assim, outro ponto será obtido com as argilas $\mathrm{A}$ e $\mathrm{C}$.

Combinação A_C

$$
\mathrm{X}_{\mathrm{A}}^{\prime}=0,50 ; \mathrm{X}_{\mathrm{C}}{ }^{\prime}=0,50, \mathrm{X}_{\mathrm{A}}{ }^{\prime}=50 \% ; \mathrm{X}_{\mathrm{C}}=50 \%
$$

Tabela VI - Dados calculados para composição de massas no exemplo.

[Table VI - Calculated values for mass composition of the example.]

\begin{tabular}{cccc}
\hline $\begin{array}{c}\text { AAm } \\
(\%)\end{array}$ & $\mathrm{X}_{\mathrm{A}}$ & $\mathrm{X}_{\mathrm{B}}$ & $\mathrm{X}_{\mathrm{C}}$ \\
\hline \multirow{2}{*}{6,0} & 0,71 & 0,29 & 0 \\
& 0,50 & 0 & 0,50 \\
& & & \\
6,5 & 0,57 & 0,43 & 0 \\
& 0,25 & 0 & 0,75 \\
7,0 & 0,86 & 0,14 & 0 \\
& 0,75 & 0 & 0,25 \\
\hline
\end{tabular}

$X_{A}, X_{B}$ e $X_{C}$ : frações dos componentes.

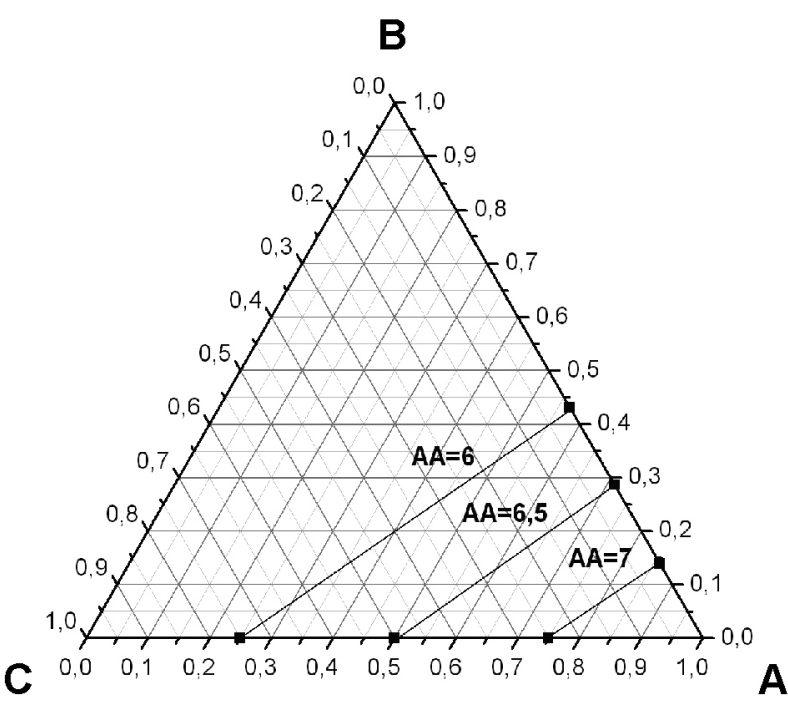

Figura 5: Gráfico de cálculo de massas para o exemplo. [Figure 5: Illustration of mass calculation for the example.] 


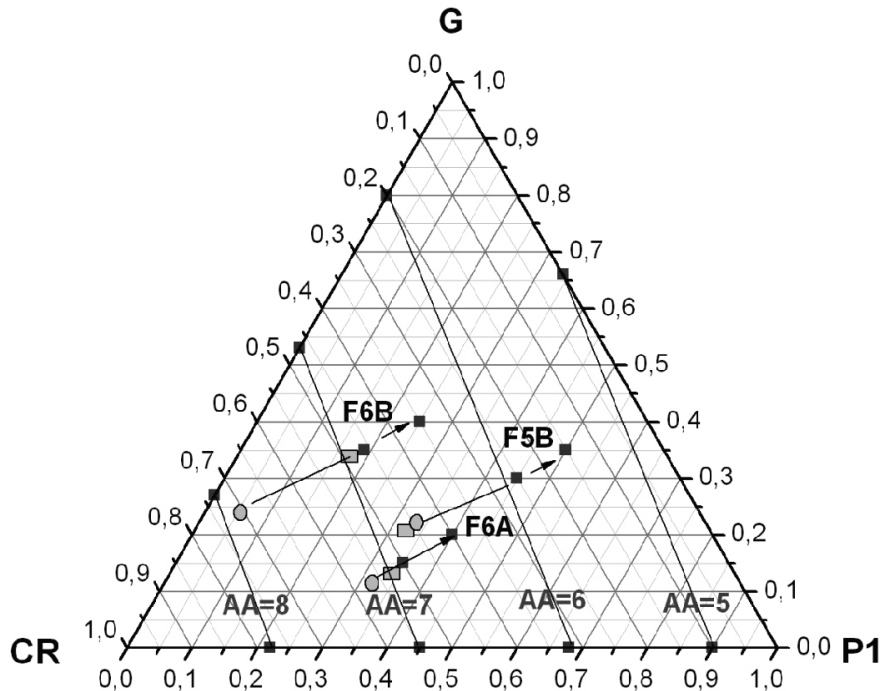

Figura 6: A figura mostra as linhas de AA constante obtida através de cálculos à temperatura de $1050^{\circ} \mathrm{C}$. Também é mostrado o valor das misturas testadas (F5B; F6A; F6B): valor calculado ( $\mathbf{\square})$, resultado experimental (o), deslocamento do ponto para fins de correção (seta) e o valor corrigido ( $\mathrm{\square}$ ).

[Figure 6: The illustration shows the lines of AA constant obtained through calculations to the temperature of $1050{ }^{\circ} \mathrm{C}$. Also the value of the tested mixtures is shown (F5B; F6A; F6B): calculated value (-), experimental result ( $(\mathrm{O})$, displacement of the point for correction (arrow) and corrected value (口)]

Desta forma serão obtidos os dados mostrados na Tabela VI e lançados em gráfico ternário (Fig. 5)

Gráficos deste tipo permitem direcionar a correção dos valores experimentais em relação aos calculados, deslocando o ponto para o componente com características que compensem as deficiências. Nas misturas testadas, os deslocamentos foram realizados em linha reta para ilustrar melhor o procedimento.

Para a realização de ensaios experimentais de formulações de três componentes foram selecionados quatro pontos (Tabela VII e Fig. 6): F5A, F5B, F6A e F6B. Observa-se que o efeito do melhor empacotamento proporcionado por $\mathrm{P} 1$, é encontrado nas amostras F5B e F6A onde está em maior concentração e onde os valores de AA são mais próximos dos calculados. As amostras F5A e

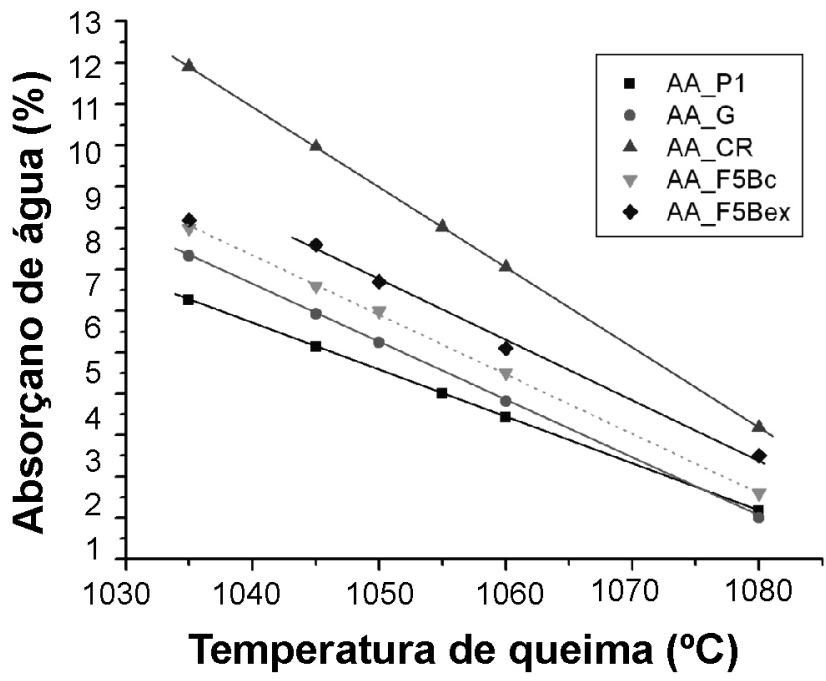

Figura 7: Variação da absorção de água com a temperatura para uma mistura de três argilas obtida com valores calculados e experimentais.

[Figure 7: Water absorption behavior with the temperature for a mixture of three clays obtained with calculated and experimental values.]

F6B, contém aproximadamente a mesma quantidade de P1, sendo que $\mathbf{G}$ e $\mathbf{C R}$ somadas perfazem aproximadamente $80 \%$ do total, prejudicando o empacotamento.

As amostras F5B, F6A e F6B foram modificadas em sua composição (aumentando aproximadamente 5\% em cada um dos componentes P1 e G e reduzindo $10 \%$ de CR), conforme mostram as setas na Fig. 5 e os dados da Tabela VIII. A maior quantidade de CR está em F6A e F6B e estas melhoraram com a redução aproximando-se ao valor desejado, enquanto F5B que já continha menos CR mostra tendência a piorar com a nova redução, sendo necessário então, um aumento de fundentes para obter $6 \%$ de AA nessa temperatura.

A evolução da composição F5B $(45 \% \mathbf{P 1 + 3 0 \% \mathbf { G } +}$ $25 \% \mathbf{C R}$ ) com o aumento da temperatura entre $1035-1080{ }^{\circ} \mathrm{C}$ é mostrada na Fig. 7, onde se observa que a diferença entre os valores esperados e os experimentais é constante ocorrendo uma aproximação entre $\mathrm{AA}_{\mathrm{CR}}$ e os valores experimentais da mistura conforme aumenta a temperatura.

Para fins de comparação com a variação apresentada

Tabela VII - Resultados experimentais $\left(1050{ }^{\circ} \mathrm{C}\right)$ das misturas ternárias selecionadas na Fig. 5 e os mesmos após a correção das proporções.

[Table VII - Experimental results $\left(1050^{\circ} \mathrm{C}\right)$ of the ternary mixtures selected in Fig. 5 and the results after modifying their proportions.]

\begin{tabular}{cccccc}
\hline Amostra & $\begin{array}{c}\text { Composição } \\
(\%)\end{array}$ & $\begin{array}{c}\text { AAcal }^{\mathrm{a}} \\
(\%)\end{array}$ & $\begin{array}{c}\mathrm{AAex}^{\mathrm{b}} \\
(\%)\end{array}$ & $\begin{array}{c}\text { Nova Composição } \\
(\%)\end{array}$ & $\begin{array}{c}\text { AAex } \\
(\%)\end{array}$ \\
\hline F5B & 45P1_30G_25CR & 6,0 & 6,7 & 50P1_35G_15CR & 6,8 \\
F6A & 35P1_15G_50CR & 7,0 & 7,2 & 40P1_20G_40CR & 7,0 \\
F6B & 19P1_35G_46CR & 7,0 & 7,9 & 25P1_40G_35CR & 6,9 \\
\hline
\end{tabular}

${ }^{a}$ absorção de água calculada, ${ }^{b}$ absorção de água experimental. 
Tabela VIII - Absorção de água calculada e experimental para mistura ternária em diferentes temperaturas.

[Table VIII - Calculated and experimental water absorption for ternary mixtures.]

\begin{tabular}{ccc}
\hline Am/ & AA $^{\mathrm{a}}$ & AA $^{\mathrm{b}}$ \\
\hline F5B_1035 & 8,0 & 8,2 \\
F5B_1045 & 6,6 & 7,6 \\
F5B_1050 & 6,0 & 6,7 \\
F5B_1060 & 4,5 & 5,1 \\
F5B_1080 & 1,6 & 2,5 \\
\hline
\end{tabular}

a absorção de água calculada para F5B em diferentes temperaturas, ${ }^{b}$ absorção de água obtida experimentalmente.

pela composição binária P1_CR (Fig. 4 ) considere-se (CR + G) como componente único com a diferença de que $\mathbf{G}$ introduziu maior quantidade de finos em relação a $\mathbf{C R}$ e com a característica de que estas partículas finas contêm mais elementos fundentes do que P1, desta forma, embora a proporção de $\mathbf{P 1}$ seja maior na composição ternária que na binária, nesta a sinterização foi melhor.

O efeito da distribuição granulométrica sobre 0 empacotamento pode ser visto comparando as duas misturas (Fig. 8) em que foi analisada a variação de AA com a temperatura, respectivamente F4R e F5B. A mistura ternária, F5B, tem maior quantidade de finos, menor de intermediários e pouco menos de grossos, lembrando que estes, por ser de maior tamanho, a diferença em peso representa pouco em termos de número de grãos.

Da mesma forma, a movimentação dos pontos no diagrama ternário, levou a um aumento de grãos finos conforme se observa na Fig. 9 onde são representadas as

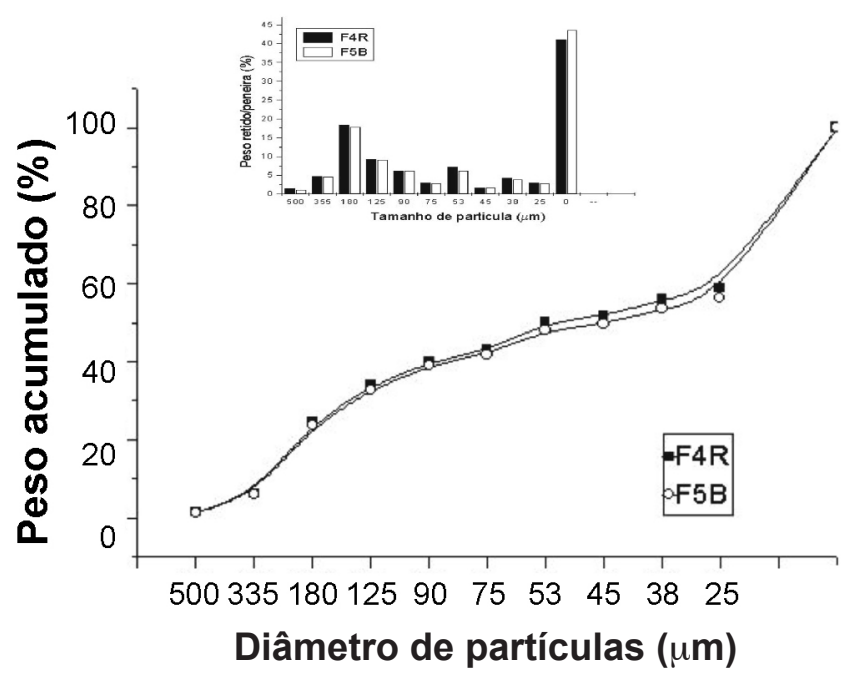

Figura 8: Distribuição granulométrica da mistura binária F4R e ternária F5B.

[Figure 8: Granulometric distribution of the binary mixture F4R and ternary F5B.] proporções granulométricas iniciais das misturas e as respectivas proporções após o deslocamento. A amostra F5B que não apresentou melhoria no ajuste da AA é a que tem maior quantidade de finos provenientes da amostra com menos fundentes ( $45 \%$ de P1), isto levou a uma densificação menor que nas outras amostras que contém maior proporção de $\mathbf{G}$ e $\mathbf{C R}$ (mais fundentes), as quais apresentaram valores satisfatórios (Tabela VI).

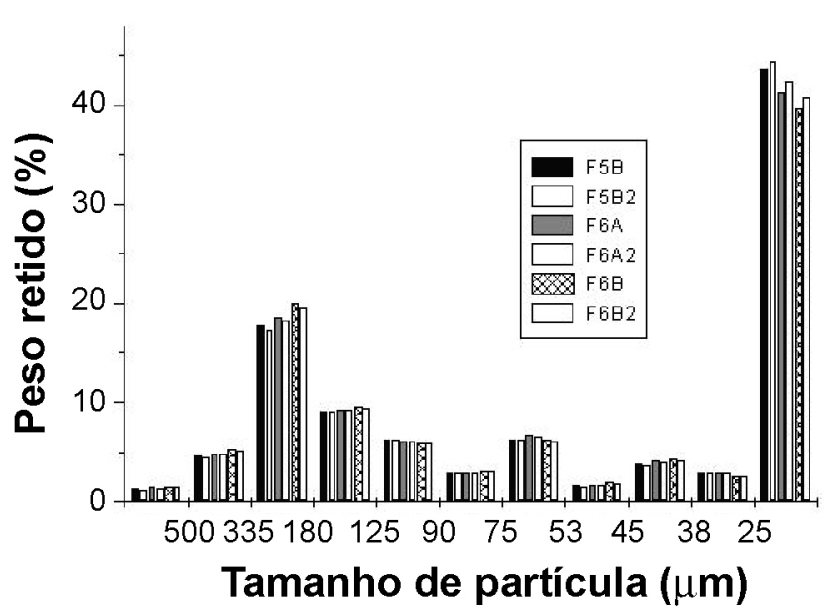

Figura 9: Distribuição granulométrica das misturas ternárias antes e depois da correção da mistura.

[Figure 9: Granulometric distribution of the ternary mixtures before and after the correction.]

\section{CONCLUSÕES}

Em ciclos de queima rápida, o início efetivo da sinterização depende principalmente do empacotamento das partículas cujos pontos de contato viabilizam a difusão sólida, por tal motivo as amostras $\mathbf{P 1}$ e $\mathbf{G}$, apesar de diferentes, apresentam comportamento de queima mais próximo. A distribuição granulométrica de $\mathbf{G}$, com maior teor de finos que $\mathbf{C R}$, produz um efeito sinterizante mais eficiente, uma vez que as reações têm início na superfície dos grãos. A argila $\mathbf{C R}$, sendo mais dura e com maior teor de quartzo e feldspato em relação a $\mathbf{G}$, resultou com uma granulometria de moagem mais grossa retardando o início da difusão sólida e a formação de suficiente fase fluída para "molhar" os grãos e apresentar aumento progressivo das reações de sinterização via fase líquida, porém a variação da AA com a temperatura foi maior nos dois intervalos (1 e 2), devido principalmente à combinação de illita e albita que proporcionam os elementos potássio e sódio, respectivamente. As argilas P1 e P2 (do topo da formação geológica), com características menos fundentes, apresentam certa semelhança de comportamento na etapa 2 (as retas são quase paralelas) diferenciando-se principalmente na temperatura em que se da início a sinterização, atrasada em $\mathbf{P 2}$, devido a deficiências no empacotamento proporcionadas pela menor variação no tamanho das partículas. A variação 
dos valores experimentais de AA para misturas preparadas tendo como base as propriedades das argilas individuais, vai depender da taxa de densificação, que por sua vez depende do empacotamento adquirido na mistura e do teor de fundentes na mesma. A correção de uma mistura de argilas, visando obter valores mais próximos às propriedades desejadas em determinadas condições de queima, é facilitada quando se conhece o comportamento das argilas individuais (ou misturas pré-fixadas) processadas nas mesmas condições. O modelo de preparação e correção de massas apresentado se mostrou eficiente, mesmo para combinação de matériasprimas com características bastante diferentes, com a vantagem de permitir direcionar as correções de acordo com as características individuais dos componentes.

\section{AGRADECIMENTO}

À FAPESP pelo suporte à pesquisa realizada.

\section{REFERÊNCIAS}

[1] A.B.N.T., Associação Brasileira de Normas Técnicas, Placas Cerâmicas para Revestimentos, NBR 1318:1997 Características Físicas (1997) 69.

[2] A. Zanardo, C. D. Roveri, M. N. Costa, M. M. T. Moreno, Anais XLIII Cong. Bras. Geologia, SBG, Salvador, BA (2006) 355-359.

[3] C. D. Roveri, A. Zanardo, M. M. T. Moreno, A. C. A. Prado, M. R. Masson, J. F. M. Motta, E. S. Bernardes,
L. Ibrahim, Anais IX World Cong. Ceram. Tile Qual. Qualicer'06, Logui Impresión, Castellón, Espanha, 3 (2006) 185-188.

[4] A. Zanardo, M. M. T. Moreno, C. D. Roveri, M. R. Masson, E. S. Bernardes, Anais XLII Cong. Bras. Geologia, SBG, Araxá, MG (2004) S02: 344.

[5] A. Zanardo, M. M. T. Moreno, C. D. Roveri, A. C. A. Prado, M. R. Masson, J. F. M. Motta, L. Ibrahim, Anais IX World Cong. Ceram. Tile Quality - Qualicer'06, Logui Impresión, Castellón, Espanha 3 (2006) 181-184

[6] C. F. Gomes, Argilas: O que são e para que servem, Fundação Calouste Gulbenkian, Lisboa, Portugal (1986) 393-408.

[7] S. R. Christofoletti, Tese, IGCE-UNESP, Rio Claro, SP (2003) 62 e 76.

[8] M. N. de S. Costa, Tese, IGCE-UNESP, Rio Claro,SP (2006) 42.

[9] R. C. Dinauer, Managing Ed., Minerals in Soil Enviroments, Madison, Wisconsin, EUA (1977) 405.

[10] L. H. Van Vlack, Propriedades dos Materiais Cerâmicos, Edgard Blücher, S. Paulo, SP (1973) 248.

[11] J. S. Reed, Principles of Ceramics Processing, $2^{\text {nd }}$ Ed., John Wiley \& Sons, New York, EUA (1995) 596.

[12] A. C. A. Prado, Cerâmica 54, 332 (2008) 466-471.

[13] A. Barba et al., Matérias Primas para la Fabricación de Soportes de Baldosas Cerámicas, $1^{\text {a }}$ Ed., Instituto de Tecnología Cerámica, Castellón, Espanha (1997) 111, 259, 273.

(Rec. 28/08/2008, Rev. 05/02/2009, Ac. 20/03/2009) 